\title{
Case Report: Confirmation by Metagenomic Sequencing of Visceral Leishmaniasis in an Immunosuppressed Returned Traveler
}

\author{
Eloise Williams, ${ }^{1,2 \star}$ Nicole S. Isles, ${ }^{3}$ Torsten Seemann, ${ }^{3}$ Trevor Kilpatrick, ${ }^{4,5,6}$ Andrew Grigg, ${ }^{7}$ Marcel Leroi, ${ }^{1,2}$ \\ Benjamin P. Howden, ${ }^{1,3}$ and Jason C. Kwong ${ }^{1,3}$ \\ ${ }^{1}$ Department of Infectious Diseases, Austin Health, Heidelberg, Australia; ${ }^{2}$ Department of Microbiology, Austin Health, Heidelberg, Australia; \\ ${ }^{3}$ Microbiological Diagnostic Unit Public Health Laboratory, Department of Microbiology and Immunology, The University of Melbourne, Peter \\ Doherty Institute for Infection and Immunity, Parkville, Australia; ${ }^{4}$ The Florey Institute of Neuroscience and Mental Health, Parkville, Australia; \\ ${ }^{5}$ Department of Anatomy and Neuroscience, The University of Melbourne, Parkville, Australia; ${ }^{6}$ Department of Neurology, Royal Melbourne \\ Hospital, Parkville, Australia; ${ }^{7}$ Department of Clinical Haematology, Austin Health, Heidelberg, Australia
}

\begin{abstract}
There has been increased interest in using metagenomic next-generation sequencing as an unbiased approach for diagnosing infectious diseases. We describe a 61-year-old man on fingolimod therapy for multiple sclerosis with an extensive travel history who presented with 7 months of fevers, night sweats, and weight loss. Peripheral blood tests showed pancytopenia and abnormal acute phase reactants. A bone marrow aspirate showed the presence of numerous intracellular and extracellular amastigotes consistent with visceral leishmaniasis (VL). Metagenomic sequencing of the bone marrow aspirate confirmed Leishmania infantum, a species widely reported in the Mediterranean region. This correlated with acquisition of $\mathrm{VL}$ infection during the patient's most recent epidemiological exposure in southern Italy 12 months prior. This case demonstrates the potential application of metagenomic sequencing for identification and speciation of Leishmania in cases of VL; however, further assessment is required using other more readily obtained clinical samples such as blood.
\end{abstract}

\section{CASE REPORT}

A 61-year-old Australian man presented with 7 months of fevers, night sweats, fatigue, and weight loss. His most recent travel was 12 months prior, comprising a 3-week trip to southern Italy; he had traveled extensively throughout Europe and Asia in the past 10 years, including a trip to India 6 years prior. His medical history was notable for relapsing remitting multiple sclerosis (MS) managed with fingolimod for the past 6 years and recent ex-smoking.

He looked unwell and was febrile at $39.2^{\circ} \mathrm{C}$; examination showed mild non-tender hepatomegaly without palpable splenomegaly or other abnormal findings. Laboratory workup revealed pancytopenia with a hemoglobin level of $121 \mathrm{~g} / \mathrm{L}$ (reference range [RR]: $130-170 \mathrm{~g} / \mathrm{L}$ ), platelets of $130 \times 10^{9} / \mathrm{L}$ (RR: $150-400 \times 10^{9} / \mathrm{L}$ ), and a white cell count of $2.6 \times 10^{9} / \mathrm{L}$ (RR: $4-12 \times 10^{9} / \mathrm{L}$ ) with a lymphocyte count of $0.3 \times 10^{9} / \mathrm{L}$ (RR: $1.5-3.5 \times 10^{9} / \mathrm{L}$ ) and a neutrophil count of $1.7 \times 10^{9} / \mathrm{L}$ (RR: $\left.2.0-8.0 \times 10^{9} / \mathrm{L}\right)$. Blood film was nondiagnostic. Acute phase reactants were also abnormal, with an erythrocyte sedimentation rate of $45 \mathrm{~mm} /$ hour, C-reactive protein of $26 \mathrm{mg} / \mathrm{L}$ (RR: $0-5)$, albumin of $30 \mathrm{~g} / \mathrm{L}$ (RR: $35-50 \mathrm{~g} / \mathrm{L}$ ), ferritin of 3,072 $\mu \mathrm{g} / \mathrm{L}$ (RR: $30-400 \mu \mathrm{g} / \mathrm{L}$ ), and polyclonal hypergammaglobulinemia. An abdominal ultrasound showed mild hepatomegaly. HIV antigen-antibody complex was nonreactive. Lymphocyte subsets were reduced, with a $\mathrm{CD} 4^{+}$Iymphopenia of $0.23 \times$ $10^{9} / \mathrm{L}$ (RR: $0.65-2.00 \times 10^{9} / \mathrm{L}$ ) and $\mathrm{CD} 8^{+}$lymphopenia of $0.21 \times$ $10^{9} / \mathrm{L}$ (RR: $0.33-1.31 \times 10^{9} / \mathrm{L}$ ). Retrospective review since commencement of fingolimod therapy 6 years prior showed a persistent lymphopenia with a total lymphocyte count between 0.2 and $0.3 \times 10^{9} / \mathrm{L}$.

Given the pancytopenia, a bone marrow aspirate and trephine were performed with the expectation of finding an aggressive hematological malignancy. Unexpectedly, the

*Address correspondence to Eloise Williams, Department of Microbiology and Infectious Diseases, Austin Health, 145 Studley Rd., Heidelberg 3084, Australia. E-mail: eloise.williams@austin.org.au aspirate revealed numerous intracellular and extracellular Leishmania amastigotes (Figure 1A and B). Whole community shotgun metagenomic sequencing of DNA extracted from the aspirate was undertaken on the lllumina NextSeq, generating $121,364,564$ sequencing reads. After removing human genome sequences, 905,743/121,364,564 (0.7\%) nonhuman sequencing reads remained, of which 294,673/905,743 (32.5\%) were classified as Leishmania reads. No Leishmania reads were identified in the negative control sample sequenced concurrently. PCR targets for small subunit ribosomal ribonucleic acid (rRNA), kinetoplast minicircle DNA, and the conserved Leishmania infantum DNA repeat region (REPL-repeat) region of the Leishmania genome were identified in silico from the metagenomic sequencing data (see Supplemental Appendix). The species, Leishmania infantum, was confirmed by in silico reconstruction of the $h s p 70$ gene and wholegenome phylogenetic comparisons of the reconstructed draft genome sequences with publicly available Leishmania genomes (Figure 1C). This correlated with acquisition of infection during the patient's most recent epidemiological exposure in southern Italy 12 months prior, rather than the possible exposure to Leishmania donovani in India 6 years prior.

After the diagnosis of visceral leishmaniasis (VL) was confirmed and on the basis that profound CD4 lymphopenia was a likely contributing factor, fingolimod was ceased. Treatment consisted of $40 \mathrm{mg} / \mathrm{kg}$ liposomal amphotericin B, which was given over 2 months. His symptoms, pancytopenia, and inflammatory markers rapidly resolved, and he remains well 6 months after treatment cessation. The CD4 lymphopenia resolved with a count of $0.73 \times 10^{9} / \mathrm{L} 3$ months after cessation of fingolimod. His MS has remained stable, except for the subsequent development of trigeminal neuralgia without evidence of new central nervous system lesions, including at the root entry zone of the trigeminal nerve in the pons. He remains off immunomodulatory therapy.

\section{DISCUSSION}

Visceral leishmanisis is a vector-borne parasitic disease caused by obligate intracellular protozoa of the genus 


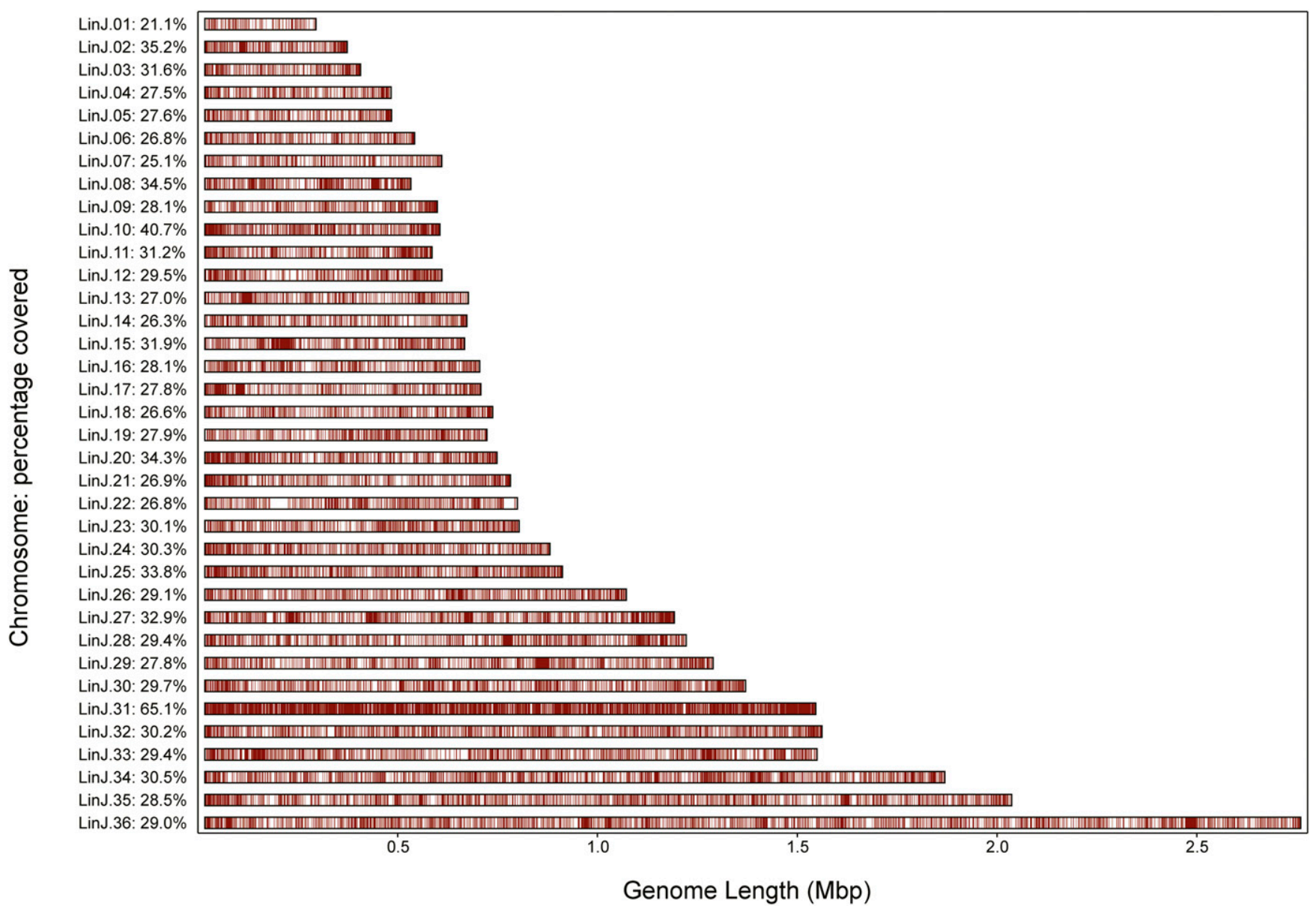

FIGURE 1. (A) Romanowsky stain of bone marrow aspirate, original magnification $\times 1,000$ : Leishman-Donovan bodies; numerous intracellular and extracellular Leishmania amastigotes are seen. (B) Romanowsky stain of bone marrow aspirate, original magnification $\times 1,000$ : Leishmania amastigotes are oval, with a round nucleus (red arrow) and rod-like kinetoplast (black arrow). (C) Maximum likelihood phylogenetic comparison of the reconstructed Leishmania genome from the patient sample (red arrow) with publicly available Leishmania genomes. The reconstructed genome was most closely grouped with Leishmania infantum. Numbers show branch support from 1,000 bootstrap replicates.

Leishmania. ${ }^{1}$ Visceral leishmaniasis is transmitted between mammalian hosts by $1.5-2 \mathrm{~mm}$ female phlebotomine sand flies, and is typically caused by $L$. donovani in Asia and Eastern Africa and $L$. infantum in Latin America, Central Asia, and the Mediterranean. The incubation period is between 2 weeks and 8 months; however, symptoms may occur years later in infected persons after they become immunosuppressed. ${ }^{1}$

In immunocompetent hosts, Leishmania-specific immune response is associated with a T-helper 1 cytokine response, including interleukin (IL)-12, interferon (IFN) gamma, and tumor necrosis factor alpha (TNF- $\alpha$ ) secretion, thereby recruiting and activating macrophages that phagocytose cells infected with leishmanial amastigotes. ${ }^{2}$ It is not surprising, therefore, that T-cell immune deficiency is known to be a risk factor for VL, with patients with HIV having 100-2,320 times the risk of developing VL compared with immunocompetent hosts. ${ }^{3}$ Solid organ transplantation, hematological and solid organ malignancies, and TNF- $\alpha$ antagonist therapy have also been associated with an increased risk of VL. ${ }^{4}$ In addition to increasing the risk of $\mathrm{VL}$, immunosuppression is also associated with reduced response rates to $\mathrm{VL}$ treatment and an increased risk of relapse. ${ }^{4}$ Treatment recommendations therefore differ for immunocompromised persons, with a higher total dose (40 mg/kg versus $21 \mathrm{mg} / \mathrm{kg}$ ) and a longer treatment duration of liposomal amphotericin B recommended in these patients. ${ }^{4}$

To our knowledge, this is only the second reported case of $\mathrm{VL}$ in the context of fingolimod therapy for MS. ${ }^{5}$ Fingolimod is a sphinogosine-1-phosphate $(\mathrm{S} 1 \mathrm{P})$ receptor inhibitor which entraps lymphocytes within lymph nodes, reducing infiltration of aggressive lymphocytes into the central nervous system. ${ }^{6}$ It was approved for the management of MS in 2009 and is often used as first-line therapy. It preferentially entraps $\mathrm{C}-\mathrm{C}$ receptor 7 (CCR7)-expressing naive and central memory lymphocytes within lymph nodes, without significantly effecting circulation of CCR7-effector memory T cells. ${ }^{7}$ Fingolimod treatment results in persistent peripheral blood lymphopenia involving both $\mathrm{CD}^{+}$and $\mathrm{CD}^{+}$cells, with a total lymphocyte count reduction of $73 \%$ compared with baseline noted after the first month of therapy in phase 3 trials. $^{6}$

Despite its lymphopenic effects, fingolimod has not been reported to significantly increase infections compared with control populations in phase 3 trials, attributed to the apparent absence of the effect of fingolimod on effector memory $T$ cells. However, review of clinical trial and post-marketing surveillance data has demonstrated an increased risk of varicella zoster virus (VZV) infection, ${ }^{8}$ John Cunningham virus causing progressive multifocal leukoencephalopathy, 
and cryptococcal infection ${ }^{9}$ in fingolimod-treated patients. Further studies have also demonstrated reduced production of pro-inflammatory cytokines IFN- $\gamma$ and IL-17 by CD4 ${ }^{+}$and $\mathrm{CD}^{+}{ }^{+}$cells ${ }^{10}$ in the presence of fingolimod. Sphinogosine-1phosphate signaling also appears to be significant in the host response to Leishmania infection, with inhibition of S1P associated with increased IL-10 production and increased intracellular parasite burden. ${ }^{11}$ Varicella serology is required before commencement of fingolimod, and VZV vaccination should be given at least 1 month before therapy in seronegative patients. There are currently no recommendations regarding prophylaxis or monitoring for other opportunistic infectious diseases for patients on fingolimod; however, clinicians should be aware of these associations and investigate specifically for opportunistic infections in patients who present with unusual symptoms while on fingolimod.

Diagnosis of VL can be difficult in those presenting in nonendemic areas such as returned travelers, particularly in immunosuppressed patients who often present atypically. A recent review of 28 imported cases of $\mathrm{VL}$ in the United Kingdom over an 18-year period found that imported cases were more likely to occur in those who were immunosuppressed (68\%), with a rise of $\mathrm{VL}$ in immunocompromised patients because of immunomodulatory drugs for autoimmune diseases compared with previous data. Tourist travel to a VL-endemic region (57\%) and travel to the Mediterranean basin (75\%) were also risk factors. ${ }^{2}$ The median time from first symptom to diagnosis was 5 months in the immunosuppressed group, with a range of 1-120 months, demonstrating the diagnostic difficulty of VL in these patients.

A number of diagnostic methods for $\mathrm{VL}$ are available and have been reviewed in detail elsewhere, with each method having variable performance characteristics with regard to sensitivity, specificity, speed, accessibility, ease of use, cost, and level of discrimination of Leishmania species. ${ }^{12}$ In the context of this patient, for whom the differential diagnosis at presentation included both infectious and noninfectious diseases, microscopic examination of the bone marrow aspirate allowed for an early and accurate preliminary diagnosis, supported by metagenomic sequencing to confirm the diagnosis and Leishmania species.

The rationale for using metagenomic sequencing to confirm the diagnosis and Leishmania species was 2-fold. First, the Leishmania PCR available in our diagnostic laboratory uses an internal transcribed space region 1 (ITS1) target that is unable to discriminate between $L$. donovani and $L$. infantum species. PCR assays based on $h s p 70$ have the ability discriminate between these species; however, these are not available in diagnostic laboratories in Australia. Our second objective was to evaluate the application of metagenomic sequencing in the diagnosis of VL. To our knowledge, this is the first reported case where metagenomic sequencing has been used for the detection of Leishmania infection. There has been increased interest in using metagenomic sequencing as a hypothesisfree, unbiased approach for diagnosing infectious diseases, with the potential for this approach to lead to a significant paradigm shift from the current diagnostic practice of performing numerous individual tests directed at specifically targeted organisms. ${ }^{13}$ Although in this case L. infantum was detected on a bone marrow aspirate where the organisms were concentrated and apparent on microscopic examination, this proof-of-principle example suggests that metagenomic sequencing has the potential to identify Leishmania species in other more accessible clinical samples such as blood. In the context of this patient from a non-endemic area who had a number of epidemiological exposures and long period of immunosuppression, we were able to infer from the sequencing data that he most likely acquired VL during his travel to the Mediterranean basin. This has implications for the choice of potentially required second-line therapy, with pentavalent antimonials no longer recommended in $L$. donovani infection acquired in India because of drug resistance. ${ }^{1}$

Although the current costs of metagenomic sequencing are high and its sensitivity compared with PCR is so far untested, this case demonstrates that it can successfully detect Leishmania infection. It is unlikely that metagenomic sequencing will replace microscopy or PCR for the diagnosis of Leishmania in endemic countries; however, it has the potential to be used in complex cases, particularly in immunocompromised patients with systemic illness where the differential diagnosis is broad or multiple pathogens may be implicated in disease.

This case of fingolimod-associated VL in a returned traveler from Italy illustrates the need for vigilance for the emergence of rare, opportunistic infections in patients on novel immunosuppressive agents, particularly following travel to endemic areas. The application of metagenomic sequencing has the potential to identify and speciate Leishmania in cases of VL; however, further assessment is required using more readily obtained clinical samples such as blood.

Received November 8, 2019. Accepted for publication July 11, 2020.

Published online September 21, 2020.

Note: Supplemental appendix and figure appear at www.ajtmh.org.

Acknowledgment: We wish to thank the Austin Health haematology laboratory for the preparation and initial interpretation of the patient's diagnostic bone marrow specimen.

Financial support: This work was supported by grants from the Austin Medical Research Foundation and the CASS Foundation (reference no. 7113). J. C. K. (GNT1142613 and Centre for Research Excellence in Emerging Infectious Diseases GNT1102962) and B. P. H. (GNT1105905) are supported by the National Health and Medical Research Council of Australia.

Disclosure: Ethics approval for metagenomic sequencing of human clinical samples was obtained through the Austin Health Human Research Ethics Committee (approval no. HREC/15/Austin/396), and informed consent was obtained from the patient as part of this protocol. Metagenomic sequence data from this study are available through the European Nucleotide Archive (BioProject PRJEBXXXXX).

Authors' addresses: Eloise Williams and Marcel Leroi, Department of Infectious Diseases, Austin Health, Heidelberg, Australia, and Department of Microbiology, Austin Health, Heidelberg, Australia, E-mails: eloise.williams@austin.org.au and marcel.leroi@austin.org. au. Nicole S. Isles and Torsten Seemann, Microbiological Diagnostic Unit Public Health Laboratory, Department of Microbiology and Immunology, The University of Melbourne, Peter Doherty Institute for Infection and Immunity, Parkville, Australia, E-mails: isles.n@ unimelb.edu.au and t.seemann@unimelb.edu.au. Trevor Kilpatrick, The Florey Institute of Neuroscience and Mental Health, Parkville, Australia, Department of Anatomy and Neuroscience, The University of Melbourne, Parkville, Australia, and Department of Neurology, Royal Melbourne Hospital, Parkville, Australia, E-mail: tkilpat@ florey.edu.au. Andrew Grigg, Department of Clinical Haematology, Austin Health, Heidelberg, Australia, E-mail: andrew.grigg@austin. org.au. Benjamin P. Howden and Jason C. Kwong, Department of Infectious Diseases, Austin Health, Heidelberg, Australia, and Microbiological Diagnostic Unit Public Health Laboratory, Department of Microbiology and Immunology, The University of Melbourne, Peter Doherty Institute for Infection and Immunity, Parkville, 
Australia, E-mails: bhowden@unimelb.edu.au and jason.kwong@ austin.org.au.

\section{REFERENCES}

1. Burza S, Croft SL, Boelaert M, 2018. Leishmaniasis. Lancet 392: 951-970.

2. Fletcher K, Issa R, Lockwood DN, 2015. Visceral leishmaniasis and immunocompromise as a risk factor for the development of visceral leishmaniasis: a changing pattern at the hospital for tropical diseases, London. PLoS One 10: e0121418.

3. Alvar J, Aparicio P, Aseffa A, Den Boer M, Cañavate C, Dedet JP, Gradoni L, Ter Horst R, López-Vélez R, Moreno J, 2008. The relationship between leishmaniasis and AIDS: the second 10 years. Clin Microbiol Rev 21: 334-359.

4. Aronson N, Herwaldt BL, Libman M, Pearson R, Lopez-Velez R, Weina P, Carvalho E, Ephros M, Jeronimo S, Magill A, 2017. Diagnosis and treatment of leishmaniasis: clinical practice guidelines by the Infectious Diseases Society of America (IDSA) and the American Society of Tropical Medicine and Hygiene (ASTMH). Am J Trop Med Hyg 96: 24-45.

5. Artemiadis AK, Nikolaou G, Kolokythopoulos D, Tegos N, Terentiou A, Triantafyllou N, Papanastasiou I, 2015. Visceral leishmaniasis infection in a fingolimod-treated multiple sclerosis patient. Mult Scler 21: 795-796.
6. Kappos L et al., 2010. A placebo-controlled trial of oral fingolimod in relapsing multiple sclerosis. $N$ Engl J Med 362: 387-401.

7. Mehling M, Brinkmann V, Antel J, Bar-Or A, Goebels N, Vedrine C, Kristofic C, Kuhle J, Lindberg RL, Kappos L, 2008. FTY720 therapy exerts differential effects on T cell subsets in multiple sclerosis. Neurology 71: 1261-1267.

8. Arvin AM et al., 2015. Varicella-zoster virus infections in patients treated with fingolimod: risk assessment and consensus recommendations for management of varicella-zoster virus infections in MS. JAMA Neurol 72: 31-39.

9. Fragoso YD, 2017. Multiple sclerosis treatment with fingolimod: profile of non-cardiologic adverse events. Acta Neurol Belg 117: 821-827.

10. Serpero LD, Filaci G, Parodi A, Battaglia F, Kalli F, Brogi D, Mancardi GL, Uccelli A, Fenoglio D, 2013. Fingolimod modulates peripheral effector and regulatory T cells in MS patients. J Neuroimmune Pharmacol 8: 1106-1113.

11. Arish M, Husein A, Ali R, Tabrez S, Naz F, Ahmad MZ, Rub A, 2018. Sphingosine-1-phosphate signaling in Leishmania donavani infection in macrophages. PLoS Negl Trop Dis 12: e0006647.

12. Akhoundi $\mathrm{M}$ et al., 2017. Leishmania infections: molecular targets and diagnosis. Mol Aspects Med 57: 1-29.

13. Forbes JD, Knox NC, Ronholm J, Pagotto F, Reimer A, 2017. Metagenomics: the next culture-independent game changer. Front Microbiol 8: 1069. 